\title{
A Jointly Optimized Variable M-QAM and Power Allocation Scheme for Image Transmission
}

\author{
Mohamed El-Tarhuni, ${ }^{1}$ Mohamed Hassan, ${ }^{1}$ and Akram Bin Sediq ${ }^{2}$ \\ ${ }^{1}$ American University of Sharjah, P.O. Box 26666, Sharjah, UAE \\ ${ }^{2}$ Department of Systems and Computer Engineering Carleton University, Ottawa, Ontario, Canada K1S 5B6
}

Correspondence should be addressed to Mohamed Hassan, mshassan@aus.edu

Received 28 March 2011; Accepted 14 November 2011

Academic Editor: Khoa Le

Copyright (C) 2012 Mohamed El-Tarhuni et al. This is an open access article distributed under the Creative Commons Attribution License, which permits unrestricted use, distribution, and reproduction in any medium, provided the original work is properly cited.

\begin{abstract}
We introduce an improved image transmission scheme over wireless channels with flat Rayleigh fading. The proposed scheme jointly optimizes bit power and modulation level to maximize the peak signal-to-noise ratio (PSNR) of the reconstructed image and hence improves the perceptual quality of the received image. In this optimization process, the significance of bits with regard to the overall quality of the image is exploited. The optimality of the proposed algorithm is demonstrated using the Lagrange method and verified through an iterative offline exhaustive search algorithm. For practical implementation, a look-up table is used at the transmitter for assigning the bit power and modulation level to each bit stream according to the received signal-to-noise ratio (SNR) observed at the receiver. The proposed scheme has low complexity since the look-up table is computed offline, only once, and used for any image which makes it suitable for devices with limited processing capability. Analytical and simulation results show that the proposed scheme with jointly optimized bit power and variable modulation level provides an improvement in PSNR of about 10 to $20 \mathrm{~dB}$ over fixed power fixed modulation (16-QAM). A further reduction in complexity is achieved by using the average signal-to-noise ratio rather than the instantaneous SNR in selecting the system parameters.
\end{abstract}

\section{Introduction}

Recent advances in wireless communications have made it more possible to transmit multimedia traffic (voice, image, video, and data) over high-speed wireless links [1, 2]. This is expected to attract more users of wireless networks with diverse applications such as video on demand, mobile TV, and medical services. Such applications, however, pose many challenges to system design since they are both power- and bandwidth-hungry applications with strict time requirements. It is therefore imperative to develop new transmission schemes that use radio resources as efficient as possible, especially for mobile users. A key point in the design of multimedia systems is how to tailor the transmission and reception schemes according to the type of traffic being sent and the dynamics of the wireless medium. Several alternatives, which can be employed either separately or jointly, have been investigated in the past, such as channel coding, source coding, power and rate control, and adaptive modulation.

Channel coding is an essential constituent of any digital communication system since it allows the system to use less signal power to achieve a target bit error rate (BER). However, channel coding results in an increase in the required bandwidth which consequently results in a reduction in the effective transmission rate. One way to reduce the loss in bandwidth efficiency due to channel coding is by using data partitioning and layered coding in association with unequal error protection (UEP) [3]. In UEP, the coded image information is usually divided into two or more partitions. For instance, the first partition typically contains the DC components and protected with a low code rate followed by one or more partitions containing the AC components that are coded at higher code rates [4-7]. A variation of the channel coding approach is joint source/channel coding (JSCC) 
[8-13] to achieve significant performance improvement, in contrast to Shannon's information separation theorem [14].

The quality of wireless channels varies due to timevarying path-loss and multipath fading [15]. However, significant improvements can be achieved through opportunistic methods that adapt to varying channel conditions such as power control [16] and rate control [17]. For instance, less transmit power is used when the channel is good while more power is used if the channel is in deep fade. Another algorithm that can be used is to use higher data rates for good channels and lower or even cease transmission when the channel is bad. In [18], a quasioptimal power allocation scheme for multimedia communications over CDMA systems was suggested. Recently, joint power allocation and source coding were proposed in [19] for image transmission over flat Rayleigh fading channels, and a robust channelbased source coder was developed. A joint power allocation and error control scheme for video transmission was also proposed in [20]. Finally, some work was done to jointly optimize the power allocation and allocated bandwidth among users in [21]. These systems require a reliable and fast feedback link from the receiver side about the channel status to allow the transmitter to adjust its parameters (power level, data rate, etc.) according to the channel conditions.

Another approach for improving the quality and data rate of wireless links is to jointly optimize the modulation scheme with some of the aforementioned techniques. For instance, a combined source coding and modulation scheme for image and video transmission was presented in [22]. Besides considering only additive white Gaussian noise (AWGN), the focus was mainly on source rate and modulation level, and no details were presented about bit power allocation. An integrated cross-layer design that jointly optimizes scheduling, modulation, and power control was proposed in [23]. The bit error rate was used as the basis for the modulation level optimization, and the importance of bits to link quality was not considered in that work. Furthermore, no restriction was set on the peak-to-average ratio of the transmitted signal. Another work that optimized the modulation level and transmitted power was presented in [24] with focus on diversity combining. Again, bit error rate was used as the optimization criterion, and the significance of bit importance to the message quality was not considered. Other related work where the different resources are jointly optimized under different systems and channel conditions have been suggested in [25-28].

In a recent work presented in [29], the authors proposed a power allocation algorithm based on the importance of different bits for improving image quality. It was shown that the proposed algorithm provides a gain of 3 to $4 \mathrm{~dB}$ in PSNR for image transmission over Rayleigh fading channels compared to the fixed power scenario. In this paper, we demonstrate the efficacy of the joint optimization of variable modulation and power allocation for transmission of sensitive information over wireless channels. Thus, we consider the case of uncompressed images. While this might look a simplified assumption, it is worth noting that certain applications require that transmitted and received data should be entirely identical. For instance, in telemedicine, digitized information such as CT images should not be changed through the transmission process, making diagnosis based on transmitted images as truthful as that based on original images. Any losses of image information during transmission might cause degradation in the quality of medical images. This in turn will affect the accuracy of the diagnosis. It is also worth noting that physicians involved in telemedicine are liable for their diagnosis based on transmitted images. This is another reason why differences in image quality before and after transmission should be minimal. Future work could consider the impact of joint optimization of variable modulation and adaptive power allocation on transmission of compressed images.

The advantage of the proposed algorithm is that it did not require any increase in the bandwidth. However, it suffered from an undesirable increase in the peak-toaverage power ratio (PAPR) in the transmitted signal. In this paper, we extend the work presented in [29] to higher modulation levels to jointly optimize the modulation level along with power allocation. Specifically, we develop the PSNR analytical relationships and the optimum solution under AWGN and fading conditions. Then, we present an exhaustive search algorithm to iteratively find the optimum solution. The paper then discusses practical implementation of the proposed system based on a look-up table approach. We also demonstrate the applicability of the optimization algorithm regardless of the transmitted image as well as the ability of the system to work based on average rather than instantaneous SNR feedback. Finally, simulations and analytical results to validate the developed models as well as illustrate achievable performance gains are presented and discussed.

The rest of the paper is organized as follow. Section 2 presents the details of the proposed system as well as the signal model. Section 3 describes the iterative optimization algorithm. Simulations and numerical results are presented in Section 4. Finally, the paper is concluded in Section 5.

\section{Proposed System Description}

We consider the baseband transmission system shown in Figure 1. In this system, raw images are encoded and delivered to mobile clients via a base station or an access point. Initially, a raw image is converted to its digital representation. Then, the signal is sampled, quantized, and coded into binary format and made available to transmission by the $M$-QAM system. Each sample is coded into $N$ bits. After source coding, the coded bit stream will be presented to the power allocation algorithm. The data is first demultiplexed into $N$ parallel streams, with each stream having the bits of similar importance from different samples; that is, most significant bits (MSB) are grouped together, then next MSB, and so forth. As we discuss later, a look-up table is then used to find the optimum energy per bit to be allocated to each stream. All bits in the same stream but from different samples (pixels), that is, having the same significance, will be allocated the same amount of bit energy. After that, each stream will be modulated using an $M$-QAM scheme which 


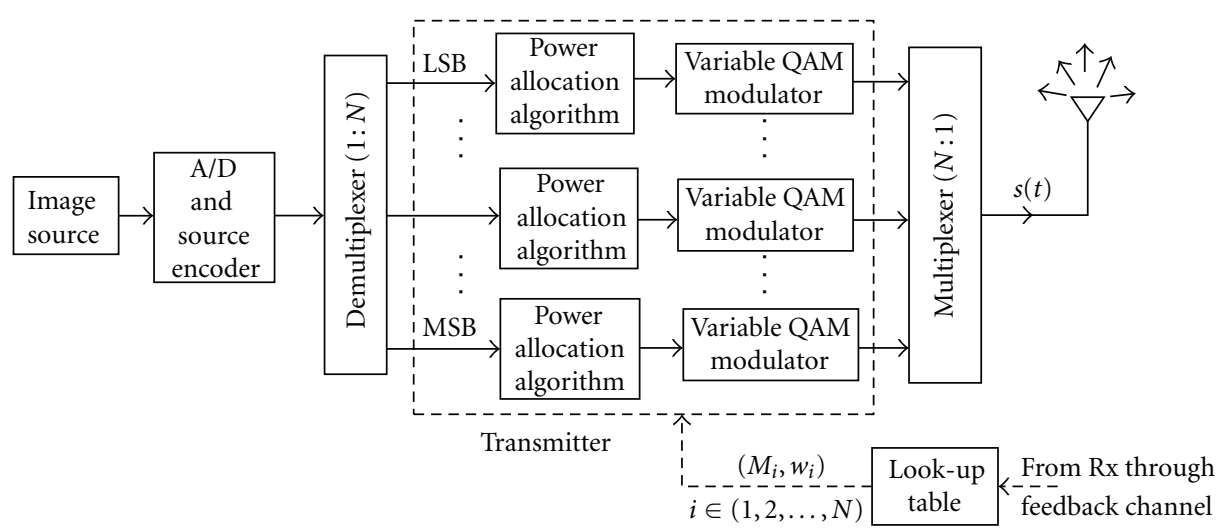

(a)

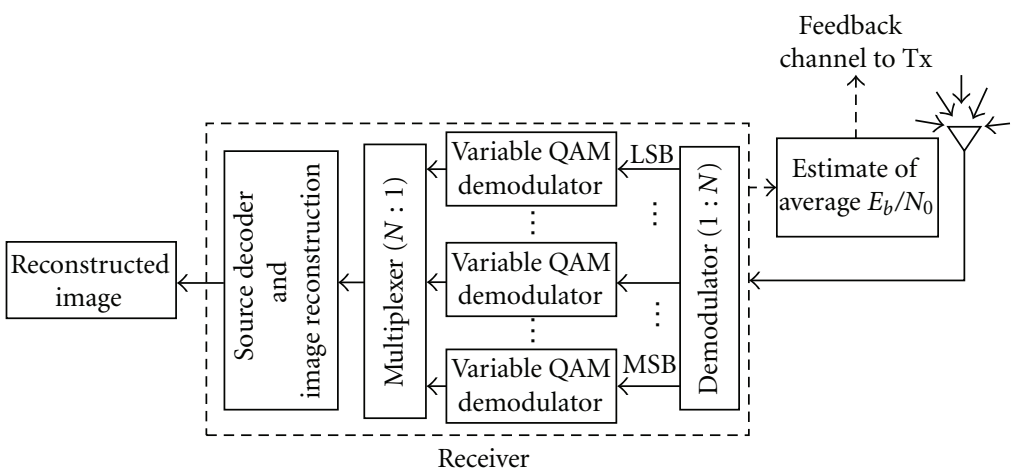

(b)

FIGURE 1: Block diagram of proposed image transmission scheme.

could either use fixed or variable modulation level. For a fixed $M$-QAM system, all streams are transmitted using the same modulation level. The only difference of the proposed scheme from a conventional $M$-QAM system is that the symbols will have different energy levels according to the significance of the bits being transmitted. In case of variable $M$-QAM, not only the symbol energy is different among the streams but also the modulation level. For instance, some streams may have low modulation level (e.g., 4-QAM) while other streams may have a higher modulation level (e.g., 16QAM) and so on. Finally, the $N$ streams are multiplexed back and sent through the channel. The baseband transmitted signal can be written as

$$
s(t)=\sum_{k=0}^{\infty} \sum_{i=0}^{N-1} \sqrt{w_{i}} g_{k i}\left(t-[k N+i] T_{b}\right),
$$

where $w_{i}$ is the transmitted power, $g_{k i}(t)$ contains information about the $i$ th symbol in the $k$ th block, and $T_{b}$ is the symbol duration. Both $g_{k i}(t)$ and the bits constituting the $i$ th symbol depend on the modulation level of the ith stream. For example, when 4-QAM is used the symbol consists of 2 bits while it consists of 6 bits when 64-QAM is used and so on. The wireless channel is modeled as a flat Rayleigh fading channel with received signal given by

$$
r(t)=\beta s(t)+n(t)
$$

where $\beta$ represents the complex channel coefficient with amplitude following the Rayleigh distribution and uniform phase over $[0,2 \pi)$. The additive white Gaussian noise (AWGN) is represented by $n(t)$ with zero-mean and twosided power spectral density of $N_{0} / 2$.

The received signal $r(t)$ is processed using a maximum likelihood detector (ML) before which the received signal is first demultiplexed into $N$ parallel streams so that bits of the same significance, for example, MSBs, and using the same modulation level are grouped together. Each stream is then detected using a ML detector to decide on the received bits. It is assumed that the detector knows the modulation level of each stream in order to detect it. To further illustrate the detection process, consider block 0 , that is, $k=0$. The sampled output of the matched filter after processing the received signal $r(t)$ at $t=(i+1) T_{b}$ is $r_{0 i}=\beta s_{0 i}+n_{0 i}$. The ML detector estimates the transmitted $M_{i}$-QAM symbol as $\hat{s}_{0 i}=x_{i \hat{j}}$ where $\hat{j}=\arg \min _{j=1, \ldots, M_{l}}\left\{\left\|r_{i}-\beta x_{i j}\right\|^{2}\right\}$ and $\left\{x_{i 0}, \ldots, x_{i\left(M_{l}-1\right)}\right\}$ is the set of $M_{i}$-QAM complex constellation points. Then, the received bits are $\Psi_{M_{i}}\left(\hat{s}_{0 i}\right)$, where $\Psi_{M_{i}}(\cdot)$ is a mapping function that maps symbol to bits in the $M_{i}$-QAM constellation. Note that the mapping function performs symbol to bits mapping such that the symbols are Gray coded (adjacent symbols differ in only one bit).

In what follows, we give the details of the proposed

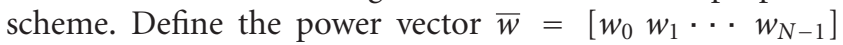


and the modulation vector $\bar{M}=\left[M_{0} M_{1} \cdots M_{N-1}\right]$. In a conventional $M$-QAM scheme, all bits carry the same amount of energy and use the same modulation level, that is, $w_{i}=w_{k}$ and $M_{i}=M_{k}$ for $i, k=0,1,2, \ldots, N-1$ and $i \neq k$. As will be shown later, this allocation is suboptimal, and we propose to optimize the allocated amount of energy and modulation level to each symbol; that is, we optimize $\bar{w}$ and $\bar{M}$ such that more protection is provided to the most important bits for the reconstructed image quality. In doing so, we keep in mind the following constraints.

(1) The average energy per bit is kept the same as that used by the conventional equal energy scheme.

(2) The bandwidth efficiency is kept greater than or equal to that of the conventional fixed modulation level scheme.

We remark that the gains achieved by optimizing power and modulation levels will be achieved at the expense of an increase in the peak-to-average power ratio (PAPR) of the transmitted signal which would result in reduction in the power efficiency of the transmitter. However, we will present an iterative offline algorithm that computes the optimum combination of power and modulation while maintaining the PAPR below a certain level. Thus, we can limit the increase in PAPR as desired. This will be discussed in the following section.

\section{Proposed Optimization Algorithm}

3.1. Optimization Criterion. Minimizing the average bit error rate (BER) during transmission is commonly a main objective when designing a communication system. The rationale behind such a choice is that minimizing the BER will result in better quality of signal transmission. This is not typically the case for image and video communications. From a perceptual quality perspective, the bits used to represent an image or a video frame do not carry the same level of importance. Hence, when errors occur in more important bits the quality of the reconstructed image will be severely degraded when compared to the case of errors happening in less important bits. Thus, the BER is a good quality measure only when all bits have the same importance. A better performance measure in such scenarios would be the peak signal-to-noise ratio (PSNR) of the reconstructed image rather than the BER. The PSNR is an objective quality metric that is most commonly used to assess the quality of reconstructed images and video sequences when transmitted over lossy channels and/or when lossy compression algorithms are involved. The PSNR is defined using the mean square error (MSE) between the original and reconstructed images. We argue that the MSE or alternatively the PSNR is better quality measure than the BER. To assert this argument, consider Figure 2 from a previous work [29]. In [29], we proposed a power allocation algorithm for image transmission over wireless channels. While the reconstructed image in Figure 2(a) has lower BER than the reconstructed image in Figure 2(b), obviously, Figure 2(a) has a worse perceptual quality when compared to Figure 2(b). This is actually a result of the fact that the MSE of the image in Figure 2(b) is lower than the MSE value of which for Figure 2(a). This also means that the PSNR of the image in Figure 2(b) is higher than the PSNR for the image in Figure 2(a).

It is important to note that the MSE is related to the BER through the mean of the received SNR which in turn affects the achieved PSNR. Also note that from the receiver perspective acceptable perceptual quality of the reconstructed image is the most essential factor. Since the PSNR is the commonly accepted objective quality metric in the literature, it is more natural to optimize the PSNR and use it as the quality metric. Therefore, we propose to minimize the MSE (maximize PSNR) rather than the BER in order to achieve better quality of received images. In this process, we need to establish a relationship between the MSE and BER for those applications. For a system with $N$ bits per sample, there are $2^{N}$ possible sequences to be transmitted. The binary representation of sample $x_{j}$ is given by the $j$ th row of the following $2^{N} \times N$ matrix:

$$
H=\left[\begin{array}{ccccc}
0 & 0 & 0 & \cdots & 0 \\
0 & 0 & \vdots & \vdots & 1 \\
\vdots & \vdots & \ddots & \vdots & \vdots \\
1 & 1 & 1 & \cdots & 0 \\
1 & 1 & 1 & \cdots & 1
\end{array}\right]
$$

with elements $h_{j k}$. The MSE in the received signal is given by

$$
\operatorname{MSE}=\frac{1}{2^{N}} \sum_{j=0}^{2^{N}-1}\left(x_{j}-\hat{x}_{j}\right)^{2} P\left(x_{j}\right),
$$

where $\hat{x}_{j}$ is the estimate of the $j$ th sample reconstructed after detecting the $N$ bits and is used to represent that sample. $P\left(x_{j}\right)$ is the a priori probability that the $j$ th sample is transmitted. Without loss of generality, consider the transmission of a zero-valued sample (i.e., $00 \cdots 000$, which is the first row in (3)). Assume that this sample was received in error. Then, the possibly received $N$-bit sequence is one of the other $2^{N}-1$ sequences in (3) other than $00 \cdots 000$. The probability that the $i$ th sample with a decimal value $i$ is reconstructed is given by

$$
\begin{array}{r}
P S_{i}=\prod_{k=0}^{N-1}\left[P_{k} \gamma_{i 0}(k)+\left(1-P_{k}\right) \bar{\gamma}_{i 0}(k)\right], \\
i=0,1,2, \ldots, 2^{N}-1,
\end{array}
$$

where $P_{k}$ is the probability that the $k$ th bit is in error and $\gamma_{i 0}(k)$ is

$$
\gamma_{i 0}(k)= \begin{cases}0 & \text { if } h_{0 k}=h_{i k} \\ 1 & \text { if } h_{0 k} \neq h_{i k}\end{cases}
$$

where $\bar{\gamma}_{i 0}(k)$ represents the complement of $\gamma_{i 0}(k)$. The MSE for the above case is calculated as

$$
\operatorname{MSE}_{0}=\frac{1}{2^{N}-1} \sum_{i=1}^{2^{N}-1} i^{2} \prod_{k=0}^{N-1}\left[P_{k} \gamma_{i 0}(k)+\left(1-P_{k}\right) \bar{\gamma}_{i 0}(k)\right] .
$$




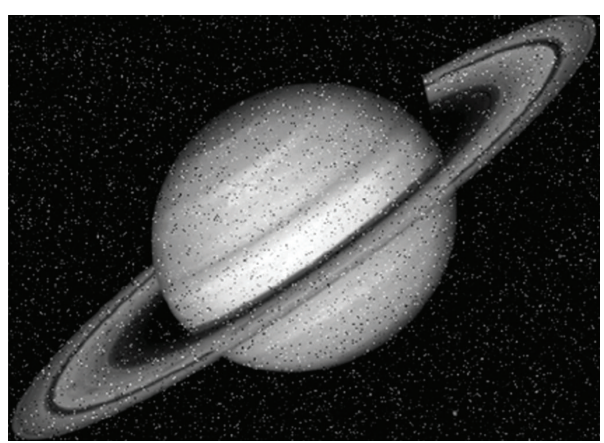

(a) $\mathrm{BER}=0.0229, \mathrm{MSE}=0.00796$

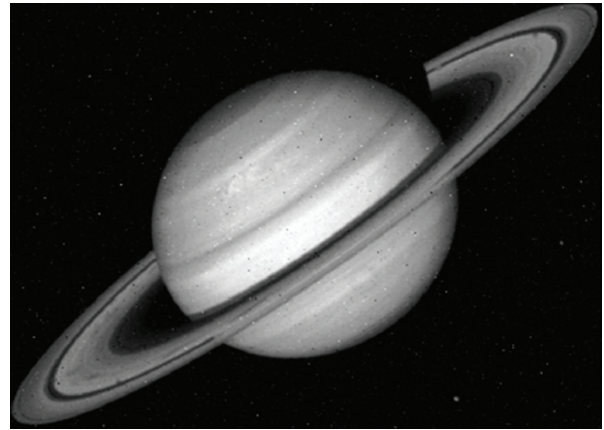

(b) $\mathrm{BER}=0.1598, \mathrm{MSE}=0.000713$

FIGURE 2: Impact of MSE and BER on the perceptual quality of reconstructed images [29].

The MSE for other samples can be obtained following a similar procedure, and the average MSE can be calculated by averaging over all possible transmitted samples. It is possible to show that, on average, all MSE values are approximately the same, and hence (7) will be the average MSE. We note that the PSNR is related to the MSE as follows:

$$
\mathrm{PSNR}=10 \log _{10} \frac{I^{2}}{\mathrm{MSE}},
$$

where $I$ is the maximum intensity value. In what follows, we will use the terms MSE and PSNR interchangeably.

Note that the probability that the $k$ th bit is in error for the $M$-QAM system in AWGN is upper bounded by

$$
P_{k} \leq a_{k} \operatorname{erfc}\left(\sqrt{b_{k} w_{k} \frac{E_{b}}{N_{0}}}\right),
$$

where "erfc" is the error complementary function, $E_{b} / N_{0}$ is the average energy per bit-to-noise power spectral density ratio, and $a_{k}$ and $b_{k}$ are parameters that depend on the $k$ th bit modulation level $M_{k}$ as follows:

$$
a_{k}=\frac{4\left(1-1 / \sqrt{M_{k}}\right)}{2 \log _{2} M_{k}}, \quad b_{k}=\frac{3 \log _{2} M_{k}}{2\left(M_{k}-1\right)} .
$$

For the $k$ th bit within the $N$ bits representing a sample, the objective is to find the combination of modulation level $M_{k}$ and the transmitted power $w_{k}$ that will result in the minimum MSE (i.e., maximum PSNR). To reduce the complexity of the optimization process, we will limit our interest to variable $M$-ary QAM modulation that can be 4QAM, 16-QAM, or 64-QAM. Hence, the total number of possible combinations to be considered for the modulations levels is $L=3^{N}$. For example all $N$ bits can use 4-QAM; $N-1$ bits with 4-QAM and one bit with 16-QAM; $N-1$ bits with 4-QAM and one bit with 64-QAM, and so forth. Even with this restriction, we notice that the number of combinations is still high. For instance, for a system with 8 bits per sample, that is, $N=8$, we need to consider 6561 possibilities. Although, the search for the optimum level is done off line (as will be explained later) it is possible to significantly reduce the number of possible combinations by imposing two conditions that ensure the maximization of bandwidth efficiency relative to fixed 16-QAM system (used as a bench mark) and adequate error protection of most significant bits as follows.

(1) The bandwidth efficiency of the considered combinations is equal to or greater than that of the conventional 16-QAM. This means that combinations with low bandwidth efficiency are excluded from the search. For example, the cases for using 4-QAM for all $N$ bits $(M=4)$; 4-QAM for $N-1$ bits and 16-QAM for one bit; 4-QAM for $N-2$ bits and 16-QAM for the two bits, and so forth will have lower bandwidth efficiencies compared with the case of 16-QAM for all bits and hence will be discarded. However, combinations with modulation levels such as 64-QAM for all $N$ bits; 64-QAM for $N-1$ bits and 4-QAM for one bit, and so forth will have higher bandwidth efficiencies compared to 16-QAM for all bits and hence will be considered in the optimization process.

(2) The modulation level assigned to most significant bits is less than or equal to that of lower significance bits, that is, $M_{i} \geq M_{j}$ where $i<j ; i, j=$ $0,1, \ldots, N-1$. This condition ensures that most significant bits (most important for image quality) have more protection against channel errors since they use a lower modulation level compared to lower significant bits.

The above two conditions will significantly reduce the number of combinations to be considered to $L_{s}$. For example, with $N=8$ only $L_{s}=25$ out of the total $L=6561$ combinations satisfy the two conditions mentioned above and hence will result in less complexity in the optimization process.

The problem now is reduced to finding the optimum power vector $\bar{w}_{\text {opt }, s}$ where $s=0,1, \ldots, L_{s}-1$ for each of these modulation level combinations as will be discussed in next section.

3.2. Optimum Solution. The problem at hand can be stated as follows. For every combination of modulation levels in the subset $L_{s}$ defined in the previous section, we would 
like to find the optimum power vector $\bar{w}_{\text {opt }, s}$ where $s=$ $0,1, \ldots, L_{s}-1$ such that the MSE is minimized subject to the constraint that the average energy per bit is kept constant. We use the Lagrange method to find a global minimum for the multivariable MSE function $f(\bar{w})$ obtained by substituting (9) in (7) subject to a constraint $y(\bar{w})=\sum_{k=0}^{N-1} w_{k}=N$. Note that this constraint guarantees that the average energy per $N$ bits is $E_{b}$; that is, the system has the same average energy as the conventional equal power scheme. As demonstrated in the Appendix, the MSE function $f(\bar{w})$ as a function of $w_{k}, k=1, \ldots, N$ is well approximated by a convex function for high $E_{b} / N_{0}$ values.

We start by finding all values of $\bar{w}$ and $\lambda$ such that

$$
\begin{aligned}
& \nabla f(\bar{w})=\lambda \nabla y(\bar{w}), \\
& \frac{\partial f(\bar{w})}{\partial w_{j}}=\lambda \frac{\partial y(\bar{w})}{\partial w_{j}}=\lambda
\end{aligned}
$$

for $j \in[0, N-1]$ where $\lambda$ is the Lagrange multiplier. The MSE function for $M$-QAM in AWGN is given by

$$
\begin{aligned}
f(\bar{w})= & \frac{1}{2^{N}-1} \sum_{i=1}^{2^{N}-1} i^{2} \\
& \times \prod_{k=0}^{N-1}\left[a_{k} \operatorname{erfc}\left(\sqrt{b_{k} w_{k} \frac{E_{b}}{N_{0}}}\right) \gamma_{i 0}(k)\right. \\
& \left.\quad\left[1-a_{k} \operatorname{erfc}\left(\sqrt{b_{k} w_{k} \frac{E_{b}}{N_{0}}}\right)\right] \bar{\gamma}_{i 0}(k)\right] .
\end{aligned}
$$

The partial derivative of the MSE function can be written as

$$
\begin{aligned}
\frac{\partial f(\bar{w})}{\partial w_{j}}= & \frac{1}{2^{N}-1} \sum_{i=1}^{2^{N}-1} i^{2} \frac{\partial}{\partial w_{j}} \\
& \times \prod_{k=0}^{N-1}\left[a_{k} \operatorname{erfc}\left(\sqrt{b_{k} w_{k} \frac{E_{b}}{N_{0}}}\right) \gamma_{i 0}(k)\right. \\
& \left.+\left[1-a_{k} \operatorname{erfc}\left(\sqrt{b_{k} w_{k} \frac{E_{b}}{N_{0}}}\right)\right] \bar{\gamma}_{i 0}(k)\right] .
\end{aligned}
$$

Using the fact that $\partial / \partial x[u \operatorname{erfc}(\sqrt{v x})]=-(u v / \sqrt{\pi v x})$ $e^{-v x}$, we can further reduce (13) into

$$
\frac{\partial f(\bar{w})}{\partial w_{j}}=\frac{1}{2^{N}-1} \sum_{i=1}^{2^{N}-1} i^{2} \prod_{k=0}^{N-1}\left[\chi_{k j} \gamma_{i 0}(k)+\bar{\chi}_{k j} \bar{\gamma}_{i 0}(k)\right]
$$

where,

$$
\begin{gathered}
\chi_{k j}= \begin{cases}-\frac{1}{\sqrt{\pi b_{k} w_{k} E_{b} / N_{0}}} a_{k} b_{k} \frac{E_{b}}{N_{0}} e^{-b_{k} w_{k} E_{b} / N_{0}} & \text { if } k=j, \\
a_{k} \operatorname{erfc}\left(\sqrt{b_{k} w_{k} \frac{E_{b}}{N_{0}}}\right) & \text { if } k \neq j,\end{cases} \\
\bar{\chi}_{k j}= \begin{cases}\frac{1}{\sqrt{\pi b_{k} w_{k} E_{b} / N_{0}}} a_{k} b_{k} \frac{E_{b}}{N_{0}} e^{-b_{k} w_{k} E_{b} / N_{0}} & \text { if } k=j, \\
1-a_{k} \operatorname{erfc}\left(\sqrt{b_{k} w_{k} \frac{E_{b}}{N_{0}}}\right) & \text { if } k \neq j .\end{cases}
\end{gathered}
$$

Substituting (14) in (11), we end up with $N$ vector equation, in addition to the constraint equation, to be solved. This vector equation is explicitly expressed as

$$
\begin{array}{r}
\frac{1}{2^{N}-1} \sum_{i=1}^{2^{N}-1} i^{2} \prod_{k=0}^{N-1}\left[\chi_{k j} \gamma_{i 0}(k)+\bar{\chi}_{k j} \bar{\gamma}_{i 0}(k)\right]=\lambda \\
\forall j \in[0, N-1] .
\end{array}
$$

The solution of the above problem results in an optimum power vector $\bar{w}_{\text {opt }}$ for a specific combination of modulation levels in the $L_{s}$ set of possible combinations. To find the global optimum power vector, the above problem is solved for each combination. Finally, a total of $L_{s}$ solutions for the optimum power vector are obtained, and only solutions that result in a PAPR less than a present threshold are used to find the global optimum solution as described below.

It is desirable to choose the optimum power vector and modulation levels that will result in the maximum PSNR and maximum bandwidth efficiency. However, some of the solutions will maximize the PSNR but with small gain in bandwidth efficiency while others may result in small gain in PSNR with higher improvement in bandwidth efficiency. This is illustrated in Table 1. Thus, there are two practical ways that can be used in making the choice:

(1) set a target fixed PSNR to be achieved and select the solution with highest bandwidth efficiency;

(2) set target fixed bandwidth efficiency and choose the solution that provides the highest PSNR.

Note that the obtained solution is valid for a particular value of $E_{b} / N_{0}$ and optimization is needed for all expected operating range of $E_{b} / N_{0}$. In other words, the optimum power vector and modulation level combination for an $E_{b} / N_{0}=0 \mathrm{~dB}$ are different from those for $E_{b} / N_{0}=5 \mathrm{~dB}$, and so forth. Finally, a look-up table that has the different values of $E_{b} / N_{0}$ and corresponding optimum vector is calculated and used for setting the transmission parameters of the bit streams. We stress that the above operation is done off line without any changes made to the look-up table during normal system operation regardless of the image to be transmitted. This can be explained by the observation that the MSE and PSNR will be the same regardless of the value of the pixel transmitted, and hence the optimum solution will 


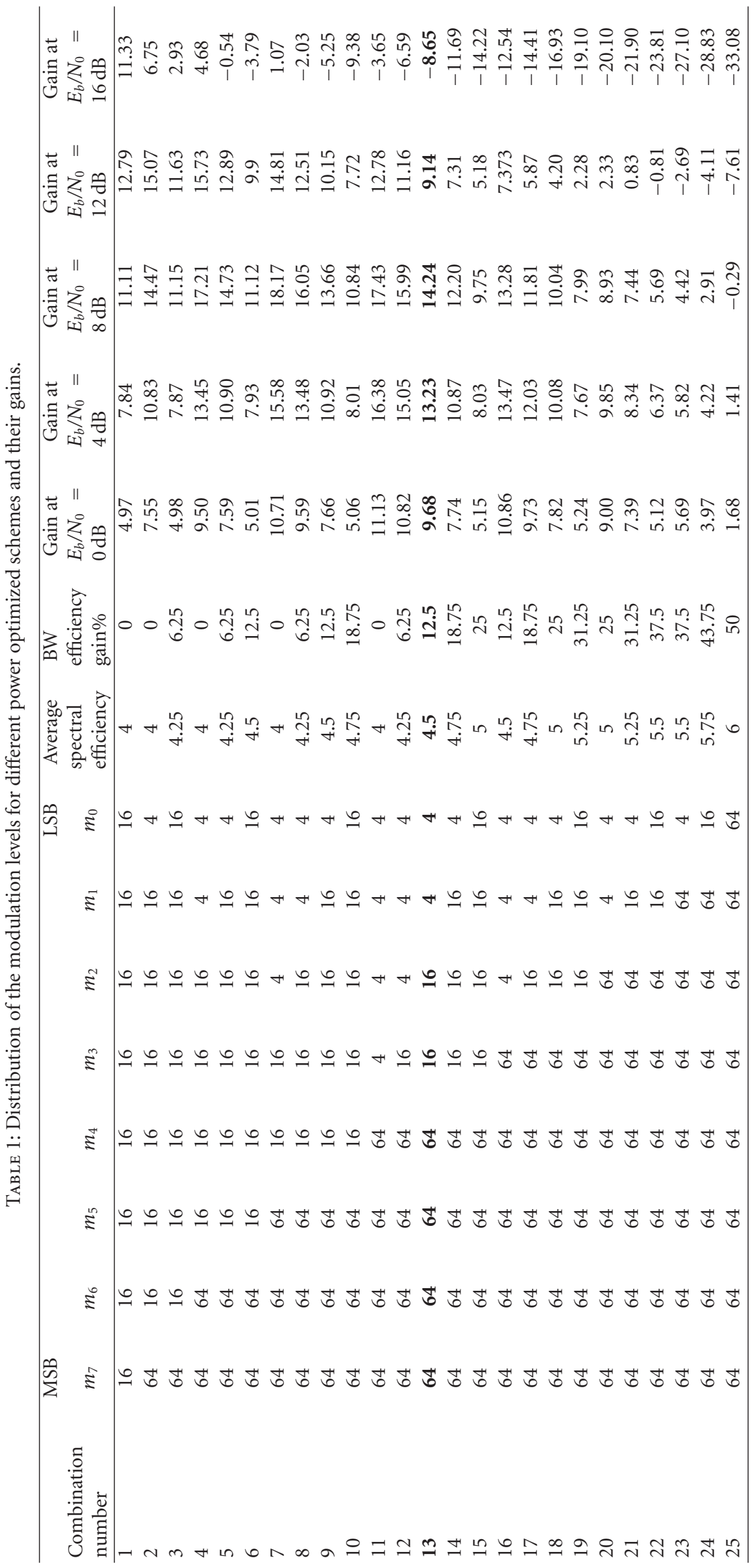


be the same for any image. However, this does not imply that the quality is the same for different images. All the sender needs to do is to get the operating $E_{b} / N_{0}$ from the receiver (through a feedback channel) and select the solution from the already stored look-up table. Since a copy of the look-up table can be prestored at the receiver, the receiver does not require any further information from the transmitter about the modulation levels. While the used modulation level can be communicated to the receiver as a preamble, recall that the receiver feeds back the average SNR to the transmitter which in turn uses it to decide the power and modulation levels. Thus, it is more natural for the receiver to maintain a lookup table similar to the one used by the transmitter. Using this look-up table, the receiver can deduce the modulation level used by the transmitter. This is expected to add a very little complexity to the receiver when compared to a conventional receiver with fixed modulation.

In the following section, we describe an iterative procedure for solving the above optimization process through an exhaustive search through all combinations of power assignment and modulation levels. We will compare the results obtained from this exhaustive search procedure with that obtained from the analytical derivation to validate their accuracy.

3.3. Exhaustive Search Algorithm. To verify the optimality of the method described in Section 3.2, a numerical iterative exhaustive search algorithm was implemented as follows.

\section{Initialization:}

(1) Initialize the power weight vector to all ones (assume the energy is the same for all bits, that is, $w_{i}=1$ for $i=0,1, \ldots, N-1)$.

(2) Calculate the MSE using (7) and (9) for a given $E_{b} / N_{0}$.

(3) Define two bits, $B$ is borrowing power bit and $D$ is donating power bit.

(4) Set the maximum limit of the PAPR to $P A P R_{\max }$ and set the energy step size to $\Delta E_{b}$.

Updating:

(1) Set $B=N-1$ (MSB as borrower) and $D=0$ (LSB as donor).

(2) Reduce the energy for the $D$ bit by $\Delta E_{b}$ and increase the energy of the $B$ bit by the same amount such that during the $n$th iteration we have

$$
\begin{aligned}
& E_{b D}(n)=E_{b D}(n-1)-\Delta E_{b} \\
& E_{b B}(n)=E_{b B}(n-1)+\Delta E_{b}
\end{aligned}
$$

Note that within a block of $N$ bits, the minimum energy per bit is zero, and the maximum energy per bit is $N E_{b}$, where $E_{b}$ is the average energy per bit.

(3) Recalculate the MSE using (7) and (9) and keep changing the energy of the two bits until you find the minimum value of MSE while the PAPR is kept less than PAPR $_{\max }$.
(4) Repeat the same procedure in (2) and (3) above with the borrower bit kept at $B=N-1$ while the donor bit $D$ is incremented by one until all other bits are used.

(5) Next, reduce the borrower bit by one to optimize the second most significant bit $(B=N-2)$ and repeat steps (2) till (4).

(6) The above steps are repeated until all bits are optimized, that is, $B=0$.

(7) During every iteration, the minimum MSE is searched for, and PAPR is ensured to be within the limit of $\mathrm{PAPR}_{\max }$. The optimum power vector is obtained after all bits have been utilized.

The above process is repeated for all modulation level combinations in the set $L_{s}$ and for every value of $E_{b} / N_{0}$ to form the look-up table as explained before. Finally, the image signal is transmitted with this optimized power and modulations levels.

It is important to make the following observations about the proposed algorithm. (a) The algorithm is implemented only once and thus the optimum power allocation can be precomputed in advance for each value of $E_{b} / N_{0}$. (b) The proposed scheme is guaranteed to converge to the combination that gives the minimum MSE because it performs an exhaustive search. Furthermore, there is no issue with convergence speed since this operation is done off line and no need to recalculate the power and modulation levels after building the look-up table.

3.4. Flat Rayleigh Fading Case. In mobile radio systems, the transmitted signal undergoes multipath propagation due to reflection, refraction, and scattering of radio waves in the wireless channel. This leads to the fluctuation of the received signal amplitude in a random manner; usually following a Rayleigh distribution for non-line-of-sight cases. The rapidly varying amplitudes result in variation of the instantaneous received signal-to-noise ratio as represented by the $E_{b} / N_{0}$. Hence, the proposed joint power and modulation level optimization needs to be modified to take into consideration the probability of error of the modulation scheme in Rayleigh fading in order to get correct estimates of the MSE and build the proper look-up table.

The best case would be obtained if the instantaneous value of the received $E_{b} / N_{0}$ is fed back to the transmitter to select the appropriate parameters (power and modulation levels) from the already constructed look-up table. However, such scenario requires not only a reliable but also a fast feedback link that is much faster than the variation in the received signal caused by the radio channel. In what follows we give an example of how fast the feedback channel should be. Consider a mobile terminal with a speed of $100 \mathrm{~km} / \mathrm{h}$ and a carrier frequency of $2 \mathrm{GHz}$. As a result, the maximum Doppler frequency $f_{D}$ is given by $f_{D}=v f_{c} / c=$ $\left(\left(100 \times 10^{3}\right) / 3600\right) \times\left(\left(2 \times 10^{9}\right) /\left(3 \times 10^{8}\right)\right)=185 \mathrm{HZ}$. Thus, the coherence time of the channel is roughly $1 / f_{D}=$ $5.5 \mathrm{~ms}$. Therefore, assuming that the $E_{b} / N_{0}$ values require 3 to 4 bits to be encoded, a feedback link with a data rate of 600 to $800 \mathrm{bps}$ is needed. We also would like to 
highlight the fact that the reliability of the feedback link could always be improved using a strong forward error correction (FEC) scheme. Obviously, the expected overhead of the needed FEC scheme will be minimal due the small size (number of bits) of the fed back information. Furthermore, to reduce the complexity and computational overhead, we construct the look-up table based on the average $E_{b} / N_{0}$ and require the receiver to send back the average $E_{b} / N_{0}$ rather than the instantaneous $E_{b} / N_{0}$. In Section 4, the simulation results show that the performance of the proposed scheme when based on the average $E_{b} / N_{0}$ is very close to the performance of which when based on the instantaneous $E_{b} / N_{0}$. In Section 4, we have also studied the sensitivity of the proposed system to possible errors in the feedback link.

To generate the look-up table for the average $E_{b} / N_{0}$-based scheme, we start by noting that the bit error probability for $M$-QAM in Rayleigh fading is given by

$P_{k} \approx \frac{1}{\log _{2} M_{k}}\left(1-\sqrt{\frac{\alpha_{k} w_{k} E_{b} / N_{0}}{1+\alpha_{k} w_{k} E_{b} / N_{0}}}\right), \quad \alpha_{k}=\frac{3 \log _{2} M_{k}}{4\left(M_{k}-1\right)}$.

Using (18) in (7) we obtain the MSE function for $M$ QAM modulation in Rayleigh fading as

$$
\begin{gathered}
f(\bar{w})=\frac{1}{2^{N}-1} \\
\quad \times \sum_{i=1}^{2^{N}-1} i^{2} \prod_{k=0}^{N-1}\left[\frac { 1 } { \operatorname { l o g } _ { 2 } M _ { k } } \left(1-\sqrt{\left.\frac{\alpha_{k} w_{k}\left(E_{b} / N_{0}\right)}{1+\alpha_{k} w_{k}\left(E_{b} / N_{0}\right)}\right) \gamma_{i 0}(k)}\right.\right. \\
+\left(\begin{array}{c}
1-\frac{1}{\log _{2} M_{k}}\left[1-\sqrt{\frac{\alpha_{k} w_{k}\left(E_{b} / N_{0}\right)}{1+\alpha_{k} w_{k}\left(E_{b} / N_{0}\right)}}\right] \\
\left.\times \bar{\gamma}_{i 0}(k)\right]
\end{array}\right.
\end{gathered}
$$

Starting from the previous expression and following the same procedure explained in Section 3.2, we end up with the same vector equation in (16) to be solved but with the following coefficients given below:

$$
\begin{aligned}
& \chi_{k j}= \begin{cases}-\frac{1}{2 \log _{2} M_{k}} \frac{\alpha_{k}}{\sqrt{\alpha_{k} E_{b} / N_{0}\left(1+\alpha_{k} w_{k} E_{b} / N_{0}\right)^{3}}} & \text { if } k=j, \\
\frac{1}{\log _{2} M_{k}}\left(1-\sqrt{\frac{\alpha_{k} w_{k} E_{b} / N_{0}}{1+\alpha_{k} w_{k} E_{b} / N_{0}}}\right) & \text { if } k \neq j,\end{cases} \\
& \bar{\chi}_{k j}= \begin{cases}\frac{1}{2 \log _{2} M_{k}} \frac{\alpha_{k}}{\sqrt{\alpha_{k} E_{b} / N_{0}\left(1+\alpha_{k} w_{k} E_{b} / N_{0}\right)^{3}}} & \text { if } k=j, \\
1-\frac{1}{\log _{2} M_{k}}\left(1-\sqrt{\frac{\alpha_{k} w_{k} E_{b} / N_{0}}{1+\alpha_{k} w_{k} E_{b} / N_{0}}}\right) & \text { if } k \neq j .\end{cases}
\end{aligned}
$$

In addition, the same exhaustive search algorithm presented in Section 3.3 can be used by employing (18) instead of (9) in step 3 in the updating part, and the input to the look-up table is the average $E_{b} / N_{0}$ rather than the instantaneous $E_{b} / N_{0}$.

\section{Analytical and Simulation Results}

In this section, we present analytical and simulation results for the PSNR obtained using the proposed scheme for both AWGN and Rayleigh fading channels. The conventional equal-power 16-QAM is used as a reference to demonstrate both the improvement in PSNR and bandwidth efficiency. The number of bits per sample (pixel) is $N=8$, and the number of modulation levels can be either 4, 16, or 64. We also investigate the degradation in PAPR compared to the conventional 16-QAM system as we optimize the power level of different bits. Initially, we consider an image transmission over AWGN channel, with $E_{b} / N_{0}=3 \mathrm{~dB}$, as shown in Figure 3. It is obvious that the proposed jointly optimized power and modulation levels result in a superior performance in terms of image quality when compared to the conventional equal power 16-QAM and the power optimized 16-QAM schemes.

The figure also shows different images transmitted using the proposed scheme without altering the look-up table. The improvement is maintained and hence supports our initial claim that the proposed optimization scheme is image independent and thus has low complexity. This low complexity results from the fact that the optimization is done in terms of the PSNR and hence is independent of the transmitted image. This means that the proposed algorithm can be used for different images with no changes. It is worth noting that the three images in Figure 3 have the same PSNRs; however, the quality of the images looks different due to the inherent limitations of PSNR as a quality measure [30]. This limitation is explained by the fact that PSNR is a fullreference metric that requires an a priori knowledge of the original image which is typically not available at the receiver side. In addition, it is known that PSNR values are not necessarily correlated with acceptable perceptual quality; that is, a reconstructed image with perceptually acceptable quality could be associated with a relatively low PSNR. Therefore, we argue that PSNR besides being full-reference metric is not enough to assess the received image quality in the presence of transmission errors.

To quantify the improvement of the proposed scheme over fixed power conventional systems, we calculate a gain parameter that indicates how much less $E_{b} / N_{0}$ the proposed scheme needs compared to the conventional system in order to achieve the same PSNR. The gain in $E_{b} / N_{0}$ achieved by the proposed power optimized 16-QAM system over the conventional equal power 16-QAM system is shown in Figure 4. Different limits on the PAPR were set to see the maximum gain achievable by the optimization process and at what expense in terms of the increase in PAPR. We see that a gain of about $2 \mathrm{~dB}$ in $E_{b} / N_{0}$ is obtained as we relax the limit in PAPR from $0 \mathrm{~dB}$ (no increase) to no limit on the PAPR where the highest gain from the optimization can be 

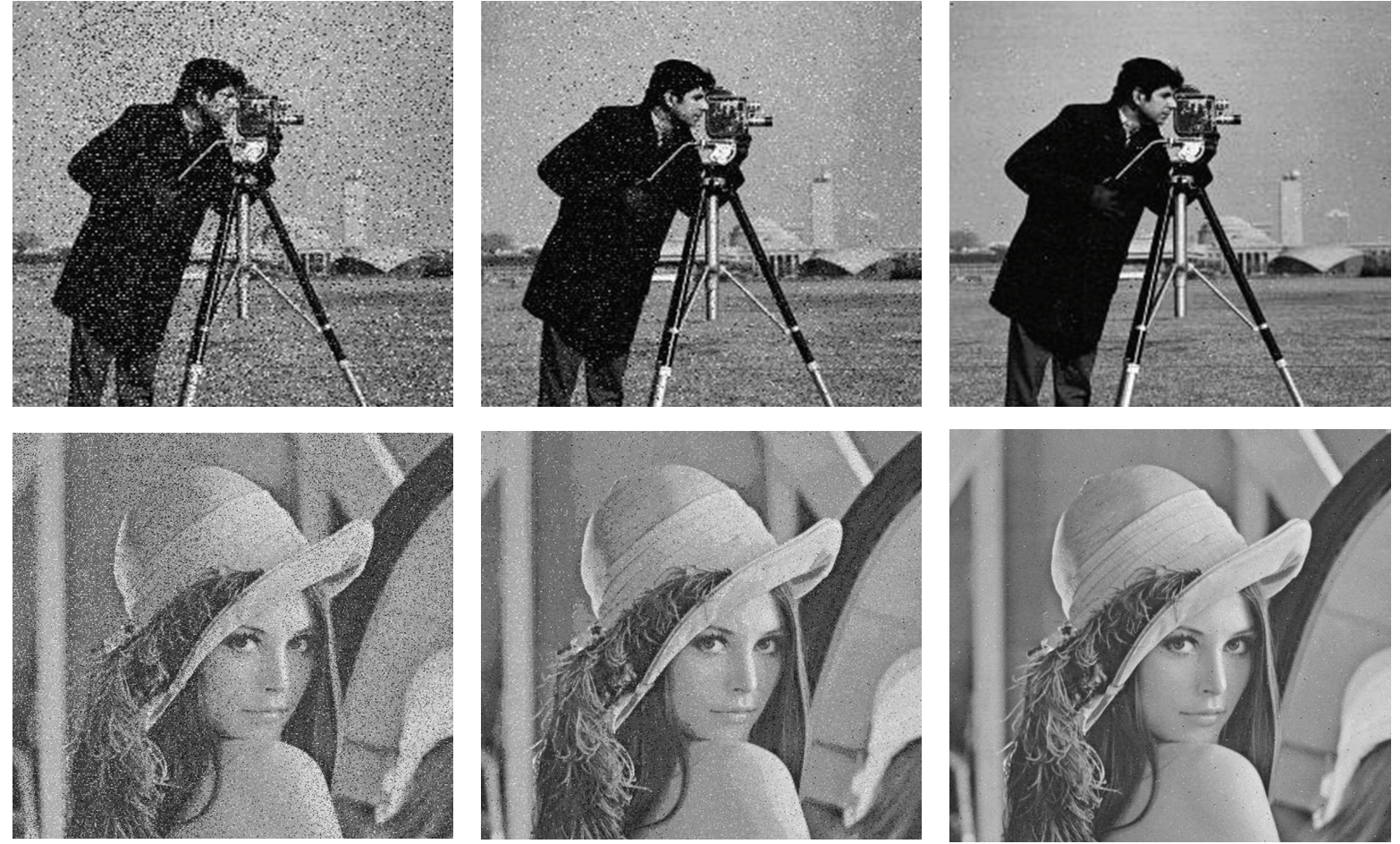

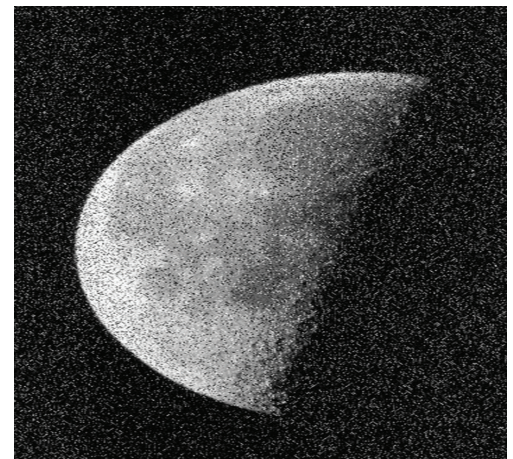

(a)

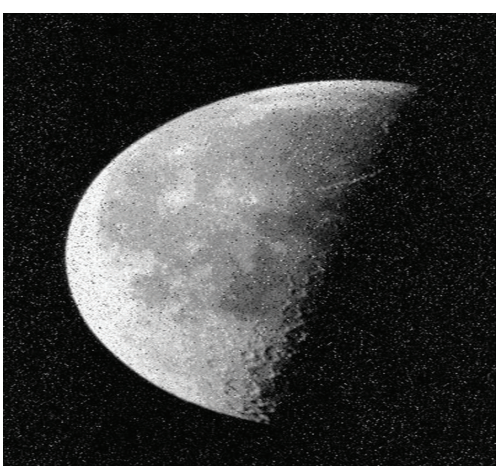

(b)

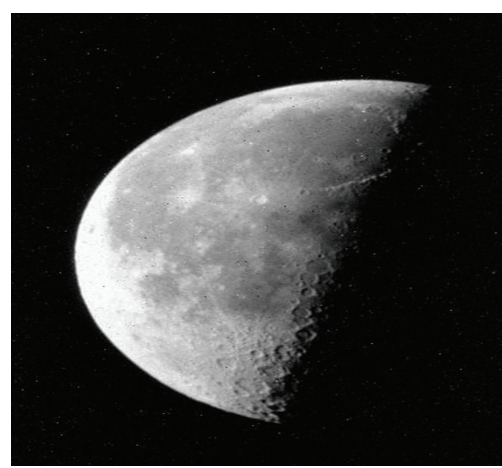

(c)

FIGURE 3: Image transmission using, (a) Conventional 16-QAM, (b) Power optimized 16-QAM, and (c) Jointly optimized power and modulation scheme.

achieved. We also note that most of the gain is achieved with an increase of about $6 \mathrm{~dB}$ in PAPR. It is observed that the gain is reduced as the PSNR increases (high $E_{b} / N_{0}$ ) and the equal power allocation scheme results in optimum performance; that is, at high $E_{b} / N_{0}$ all bits would be important and need to be equally protected.

Similar observations for the case of joint power and modulation level optimized system can be seen from Figure 5, however with much higher gain (about $6.5 \mathrm{~dB}$ ). The combination is used in this figure with modulation levels of $(64,64,64,64,16,16,4,4)$. Note that with the limit on PAPR set to $0 \mathrm{~dB}$, that is, no power optimization, the gain (about $3 \mathrm{~dB}$ ) is mainly due to variable modulation level. More gain is achieved as we relax the PAPR constraint and optimize the power as well. One other advantage of the variable $M$-QAM is the improvement in bandwidth efficiency compared to the 16-QAM system. This can be quantified by noticing that the average modulation level with optimum performances is 22.5 (from Table 1) which results in a gain of about $12.5 \%$ in bandwidth efficiency. Thus, Figures 4 and 5 indicate that most of the gain (about $6.5 \mathrm{~dB}$ in PSNR) is achieved when variable modulation is used with an increase of about $5 \mathrm{~dB}$ in PAPR. However, if the system has more conservative limit on the PAPR then a smaller gain (about $3 \mathrm{~dB}$ ) is obtained with the limit of $0 \mathrm{~dB}$ on PAPR. It is worth noting that the PAPR increases because we are changing the amplitude of the transmitted bits to optimize the quality of the reconstructed image.

Figure 6 shows the PSNR for image transmission over an AWGN channel with 8 bits per sample, $N=8$, as a function of the $E_{b} / N_{0}$. The results demonstrate the validity of the proposed optimization scheme using the Lagrange method since it has excellent agreement with the exhaustive search procedure. Both 16-QAM fixed modulation with 


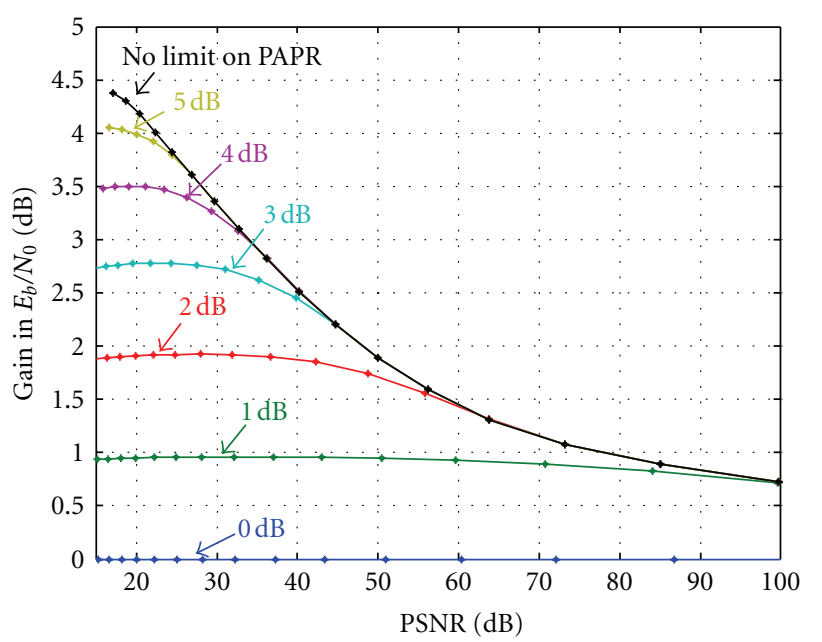

FIGURE 4: Improvement in the PSNR of power optimized 16-QAM when compared with conventional 16-QAM.

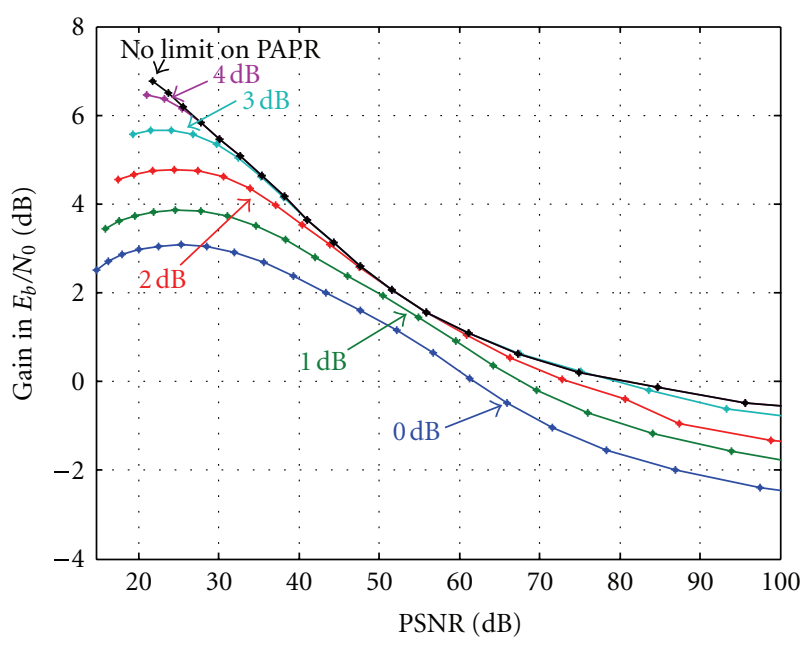

FIGURE 5: Improvement in the PSNR of power optimized variable $M$-QAM when compared with conventional 16-QAM.

optimized power only and joint power and modulation level optimization cases are presented. The PSNR for a nonoptimized 16-QAM system is also shown to demonstrate the improvement in PSNR of the proposed scheme. For instance, at $E_{b} / N_{0}=6 \mathrm{~dB}$, gains in PSNR of 10 and $20 \mathrm{~dB}$ are achieved by the power optimized 16-QAM system and the joint power and modulation level optimized system, respectively.

As we explained earlier, the proposed scheme uses a look-up table to select the best combination of power and modulation level for each bit. The only input needed for the look-up table is $E_{b} / N_{0}$. This is expected to be provided through a feedback channel from the receiver to the sender. In any real implementation, there will be some error in providing such feedback. For example, suppose the actual $E_{b} / N_{0}=3 \mathrm{~dB}$ but the feedback has an error of $+2 \mathrm{~dB}$. Then, the sender will use the entries of the look-up table for $E_{b} / N_{0}$ corresponding to $5 \mathrm{~dB}$ and there will be some

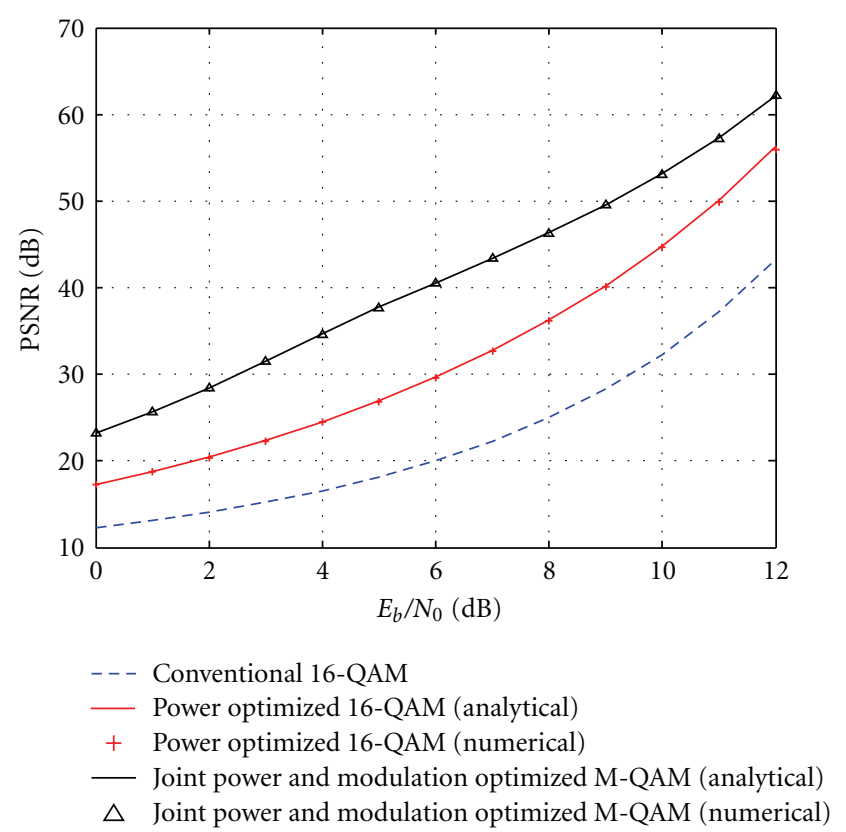

FIGURE 6: Comparison of PSNR performance in AWGN for analytical and numerical methods.

loss in performance. Figure 7 shows the sensitivity of the PSNR performance to such errors. As shown in the figure, for $E_{b} / N_{0}=3 \mathrm{~dB}$, both the power optimized and joint power and modulation level optimized schemes can tolerate an error of $\pm 5 \mathrm{~dB}$ without noticing any significant degradation in the PSNR. However, for $E_{b} / N_{0}=10 \mathrm{~dB}$, they can tolerate an error of $\pm 3 \mathrm{~dB}$. Yet, both schemes are better than the conventional scheme even with an error of $\pm 10 \mathrm{~dB}$. Figure 7 also shows that the PSNR performance of the proposed system is not very sensitive to estimation errors. It can actually tolerate errors up to $\pm 5 \mathrm{~dB}$. Note that the proposed scheme always outperforms conventional systems even in the presence of large estimation errors. This shows that the system is robust to errors in estimating $E_{b} / N_{0}$.

The PSNR performance in a flat Rayleigh fading channel is presented in Figure 8. Both Lagrange method and the exhaustive search iterative algorithm gave the same performance in fading, which proves the accuracy of the analytical optimization in the fading case. The power optimized 16QAM and the joint power and modulation level optimized schemes provide a gain in $E_{b} / N_{0}$ of about $8 \mathrm{~dB}$ and $20 \mathrm{~dB}$ over the conventional equal power 16-QAM, respectively. The figure also shows that the scheme based on feedback of average $E_{b} / N_{0}$ has a relatively small degradation in performance (about $1 \mathrm{~dB}$ in $E_{b} / N_{0}$ ) compared to the case of using instantaneous $E_{b} / N_{0}$ and the degradation vanishes at high $E_{b} / N_{0}$. This shows that we can use the average $E_{b} / N_{0}$ based scheme which has a significant reduction in complexity without paying much price in terms of PSNR performance.

Finally, we would like to clarify the fact that the online operation of the proposed scheme is distributed between the receiver and the transmitter. At one side, the scheme only requires the receiver to feedback the average SNR such 


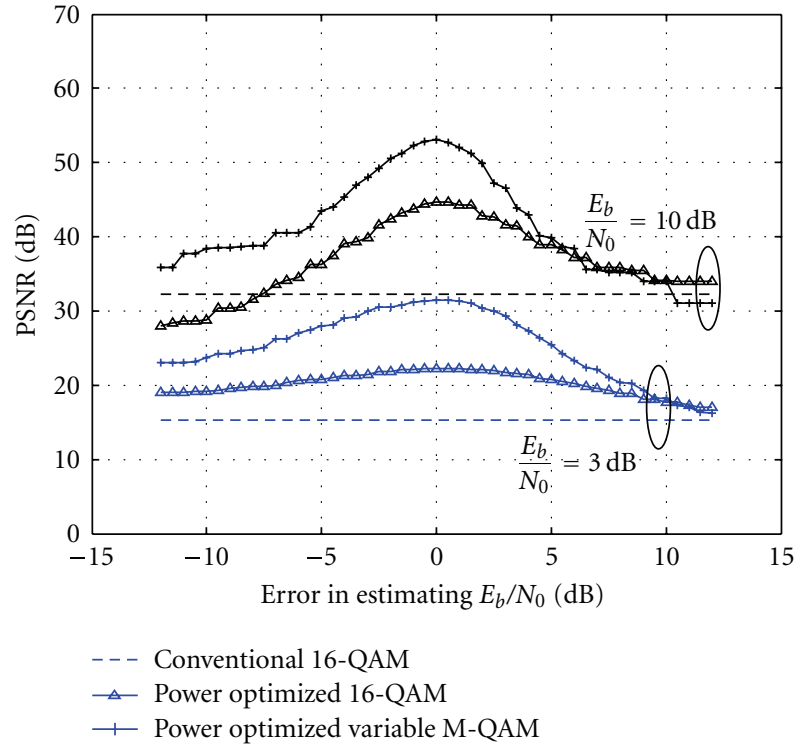

FIgURE 7: Sensitivity of PSNR to estimation error in $E_{b} / N_{0}$.

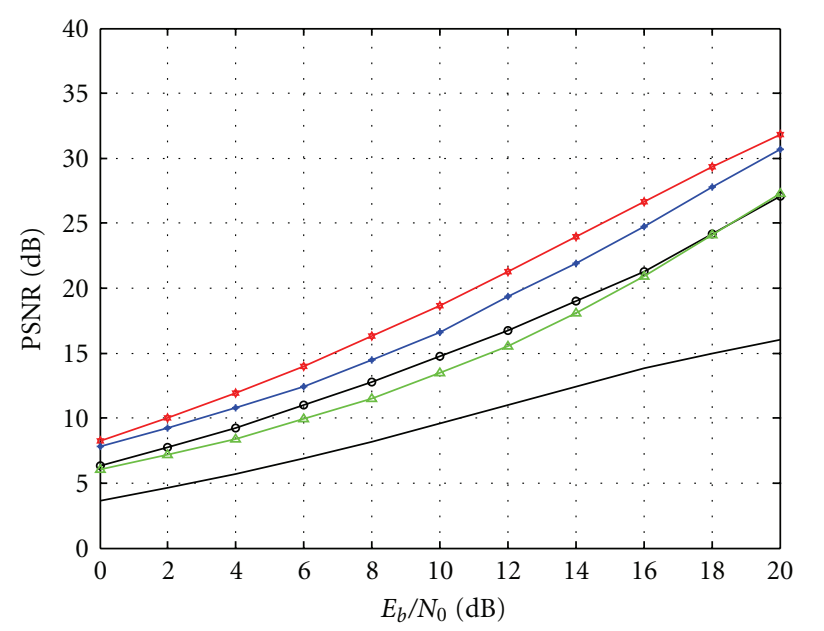

- 16-QAM conventional

$\rightarrow$ Instantaneous $E_{b} / N_{0}$-based scheme 16-QAM optimized power allocation

*- Instantaneous $E_{b} / N_{0}$-based scheme variable M-QAM with optimized power allocation

$\triangle$ Average $E_{b} / N_{0}$-based scheme 16-QAM optimized power allocation

$\rightarrow$ Average $E_{b} / N_{0}$-based scheme variable M-QAM with optimized power allocation

FIGURE 8: PSNR performance in Rayleigh fading.

that the transmitter decides the optimum combination of power allocation and modulation level for this SNR using the already built look-up table. Clearly, at the receiver side this is a minor operation with very low complexity. On the other side, the transmitter uses the value of the SNR as fed back by the receiver to look-up the optimum power allocation and modulation level which is almost of negligible complexity. The main source of complexity is the offline processing in which we build the look-up table that is used in the selection process of power and modulation levels. In this work we assumed that the transmitter is responsible for building this table. However, this operation is done only once and is independent of the image being transmitted as demonstrated in Figure 3. We also argue that the algorithm is guaranteed to converge. This is actually true because the proposed algorithm performs an exhaustive search among all possible combinations (there are 6561 possible combinations for 3 modulation levels and 8 bits per sample). Then it selects the combination that gives the highest PSNR. It is worth noting that the complexity associated with the exhaustive search could be further reduced by only searching a feasible subset among the possible combinations. As we have already shown in Section 3.1, this approach was adopted in the proposed scheme where we had to search a subset of 25 possibilities. This subset was decided to ensure that both the bandwidth efficiency and PSNR are maximized.

\section{Conclusions}

In this paper, an algorithm for joint optimization of transmitted bit power and modulation level according to the importance of the bit to the image quality was presented. The optimization was based on minimizing the mean-square error (MSE) or equivalently maximizing the peak signalto-noise ratio (PSNR) of the reconstructed image. The optimality of the proposed scheme was derived using the Lagrange method and verified through exhaustive numerical search algorithm. Improvements in PSNR of 10 to $20 \mathrm{~dB}$ were demonstrated for a modulation scheme with 4,16 , or 64 levels QAM over a conventional fixed power 16-QAM system. The proposed scheme also improved the bandwidth efficiency and hence the rate of data transmission. Finally, practical issues in terms of increase in peak to average power ratio and sensitivity of system operation to errors in $E_{b} / N_{0}$ feedback were addressed.

\section{Appendix}

\section{On the Convexity of the MSE}

In what follows, we prove that the multivariable MSE function $f(\bar{w})$, as a function of $w_{k}, k=1, \ldots, N$, is well approximated by a convex function so that we can use the Lagrange method to find the global minimum. This is actually true for high $E_{b} / N_{0}$ values where the probability that the $k$ th bit is in error denoted by $P_{k}$, is very small. As a result, the quantity $\left(1-P_{k}\right)$ is assumed to be very close to 1 . Thus using $\left(1-P_{k}\right) \approx 1,(7)$ reduces to

$$
\operatorname{MSE}_{0} \approx \frac{1}{2^{N}-1} \sum_{i=1}^{2^{N}-1} i^{2} \prod_{k=0}^{N-1}\left[P_{k} \gamma_{i 0}(k)+\bar{\gamma}_{i 0}(k)\right] .
$$

To establish the proof that the cost function is convex, we use the following properties of log-convex functions (pages: 104-106 [31]). 
(i) Log convexity is preserved under multiplication, addition, and integration.

(ii) A log-convex function is also convex.

Based on the abovementioned properties, to prove that the $\mathrm{MSE}_{0}$ is convex in $w_{k}, k=1, \ldots, N$, it is sufficient to prove that the probability $P_{k}$ is $\log$ convex in $w_{k}$. This is demonstrated for the two cases of instantaneous $E_{b} / N_{0}$-based scheme and the average $E_{b} / N_{0}$-based scheme.

Instantaneous $E_{b} / N_{0}$-based scheme.

In this scheme, using Craig's formula for the error function as given in [32], (9) which is the probability that the $k$ th bit is in error can now be written as

$$
P_{k} \leq a_{k} \frac{2}{\pi} \int_{0}^{2 \pi} e^{-\left(b_{k} w_{k}\left(E_{b} / N_{0}\right)\right) / \sin ^{2} \theta} d \theta
$$

Using the fact that log convexity is preserved under integration and since $e^{-\left(b_{k} w_{k}\left(E_{b} / N_{0}\right)\right) / \sin ^{2} \theta}$ is log convex in $w_{k}$ for all $\sin ^{2} \theta$ (page 104 [31]), as a result the quantity $a_{k} \operatorname{erfc}\left(\sqrt{b_{k} w_{k} E_{b} / N_{0}}\right)$ is also log convex in $w_{k}$.

Average $E_{b} / N_{0}$-based scheme:

In this scheme, the probability that the $k$ th bit is in error is approximated by

$$
P_{k} \approx \frac{a_{k}}{E_{b} / N_{0}} \int_{0}^{\infty} \operatorname{erfc}\left(\sqrt{b_{k} w_{k} x}\right) e^{-x /\left(E_{b} / N_{0}\right)} d x .
$$

Since $\operatorname{erfc}\left(\sqrt{b_{k} w_{k} x}\right) e^{-x /\left(E_{b} / N_{0}\right)}$ is log convex in $w_{k}$ for all $x \geq 0$ as shown above for the Instantaneous $E_{b} / N_{0}$-based scheme, $P_{k}$ is also log convex in $w_{k}$ (integration of log-convex functions). This proves the convexity of MSE in $w_{k}$ which motivated us to use the Lagrange method to find the optimal solution.

\section{References}

[1] 3GPP, "Technical specification group radio access network: physical layer-general specification," 2003, http://www .3gpp.org.

[2] GPP2 C.S0002, "Physical layer standard for CDMA2000 spread spectrum system," 2011, http://www.3gpp2.org .

[3] A. Albanese, J. Blömer, J. Edmonds, M. Luby, and M. Sudan, "Priority encoding transmission," IEEE Transactions on Information Theory, vol. 42, no. 6, pp. 1737-1744, 1996.

[4] Y. M. Targali and E. V. Jones, "Unequal error protection for image transmission over GSM networks," in Proceedings of the IEE European Workshop on Distributed Imaging, pp. 1-6, London, UK, 1999.

[5] H. Liu and M. El Zarki, "Transmission of video telephony images over wireless channels," Wireless Networks, vol. 2, no. 3, pp. 219-228, 1996.

[6] C. L. Huang and S. Liang, "Unequal error protection for MPEG-2 video transmission over wireless channels," Signal Processing: Image Communication, vol. 19, no. 1, pp. 67-79, 2004.

[7] Y. Q. Zhang, Y. J. Liu, and R. L. Pickholtz, "Layered image transmission over cellular radio channels," IEEE Transactions on Vehicular Technology, vol. 43, no. 3, pp. 786-796, 1994.

[8] M. E. Buckley, M. G. Ramos, S. S. Hemami, and S. B. Wicker, "Perceptually-based robust image transmission over wireless channels," in Proceedings of the International Conference on Image Processing (ICIP '00), pp. 128-131, September 2000.
[9] Z. Wu, A. Bilgin, and M. W. Marcellin, "Joint source/channel coding for image transmission with JPEG2000 over memoryless channels," IEEE Transactions on Image Processing, vol. 14, no. 8, pp. 1020-1032, 2005.

[10] J. Cai and C. W. Chen, "Robust joint source-channel coding for image transmission over wireless channels," IEEE Transactions on Circuits and Systems for Video Technology, vol. 10, no. 6, pp. 962-966, 2000.

[11] R. H. Morelos-Zaragoza, M. P. C. Fossorier, S. Lin, and H. Imai, "Multilevel coded modulation for unequal error protection and multistage decoding-Part I: symmetric constellations," IEEE Transactions on Communications, vol. 48, no. 2, pp. 204-213, 2000.

[12] X. Wang and M. T. Orchard, "Design of superposition coded modulation for unequal error protection," in Proceedings of the Proceedings of the IEEE International Conference on Communications (ICC'01), pp. 412-416, June 2001.

[13] T. Cover and J. Thomas, Elements of Information Theory, John Wiley \& Sons, New York, NY, USA, 1991.

[14] I. Kozintsev and K. Ramchandran, "Robust image transmission over energy-constrained time-varying channels using multiresolution joint source-channel coding," IEEE Transactions on Signal Processing, vol. 46, no. 4, pp. 1012-1026, 1998.

[15] W. C. Jakes, Microwave Mobile Communications, John Wiley \& Sons, New York, NY, USA, 1974.

[16] Q. Zhang, Z. Ji, W. Zhu, and Y. Q. Zhang, "Power-minimized bit allocation for video communication over wireless channels," IEEE Transactions on Circuits and Systems for Video Technology, vol. 12, no. 6, pp. 398-410, 2002.

[17] A. J. Goldsmith and S.-G. Chu, "Variable-rate variablepower MQAM for fading channels," IEEE Transactions on Communications, vol. 45, no. 10, pp. 1218-1230, 1997.

[18] T. Shu and Z. Niu, "Uplink capacity optimization by power allocation for multimedia CDMA networks with imperfect power control," IEEE Journal on Selected Areas in Communications, vol. 21, no. 10, pp. 1585-1594, 2003.

[19] G. H. Hakonsen, T. A. Ramstad, and A. Gjendemsj, "Image transmission with adaptive power and rate allocation over flat fading channels using joint source channel coding," Communications in Computer and Information Science, vol. 9, pp. 312-321, 2008.

[20] S. Zhao, Z. Xiong, and X. Wang, "Joint error control and power allocation for video transmission over CDMA networks with multiuser detection," IEEE Transactions on Circuits and Systems for Video Technology, vol. 12, no. 6, pp. 425-437, 2002.

[21] K. Kumaran and H. Viswanathan, "Joint power and bandwidth allocation in downlink transmission," IEEE Transactions on Wireless Communications, vol. 4, no. 3, pp. 1008-1015, 2005.

[22] H. Zheng and K. J. Liu, "The subband modulation: a joint power and rate allocation framework for subband image and video transmission," IEEE Transactions on Circuits and Systems for Video Technology, vol. 9, no. 5, pp. 823-838, 1999.

[23] W. Huang and K. Letaief, "Multilevel coded modulation for unequal error protection and multistage decodingpart I: symmetric constellations," IEEE Transactions on Communications, vol. 55, pp. 728-739, 2007.

[24] A. Gjendemsjø, H. C. Yang, G. E. Oien, and M. S. Alouini, "Joint adaptive modulation and diversity combining with downlink power control," IEEE Transactions on Vehicular Technology, vol. 57, no. 4, pp. 2145-2152, 2008.

[25] I. Pei and J. Modestino, "Multi-layered video transmission over wireless channels using an adaptive modulation and coding scheme," in Proceedings of the IEEE International 
Conference on Image Processing, vol. 2, pp. 1009-1012, October 2005.

[26] A. Babaei and B. Abolhassani, "A new iterative method for joint power and modulation adaptation in cellular systems," in Proceedings of the 2 nd IFIP International Conference on Wireless and Optical Communications Networks (WOCN '05), pp. 9497, March 2005.

[27] J. B. Kim and D. Kim, "Joint adaptive modulation and power allocation for variable-rate space-time block codes," IEEE Communications Letters, vol. 12, no. 1, pp. 26-28, 2008.

[28] Q. Du and X. Zhang, "Joint power and constellation size adaptation for mobile multicast employing M-QAM over wireless fading channels," in Proceedings of the IEEE International Conference on Communications (ICC '05), pp. 5945-5950, June 2005.

[29] A. Bin Sediq and M. El-Tarhuni, "MMSE power allocation for image and video transmission over wireless channels," in Proceedings of the IEEE 16th International Symposium on Personal, Indoor and Mobile Radio Communications (PIMRC '05), pp. 1060-1064, September 2005.

[30] M. Hassan, T. Landolsi, H. Mukhtar, and T. Shanableh, "Skip length and inter-starvation distance as a combined metric to assess the quality of transmitted video," in Proceedings of the 5th International Workshop on Video Processing and Quality Metrics for Consumer Electronics (VPQM '10), Phoenix, Ariz, USA, January 2010.

[31] S. Boyd and L. Vandenberghe, Convex Optimization, Cambridge University Press, New York, NY, USA, 2004.

[32] J. W. Craig, "A new, simple and exact result for calculating the probability of error for two-dimensional signal constellations," in Proceedings of the IEEE Military Communications Conference (MILCOM '91), vol. 2, pp. 571-575, November 1991. 

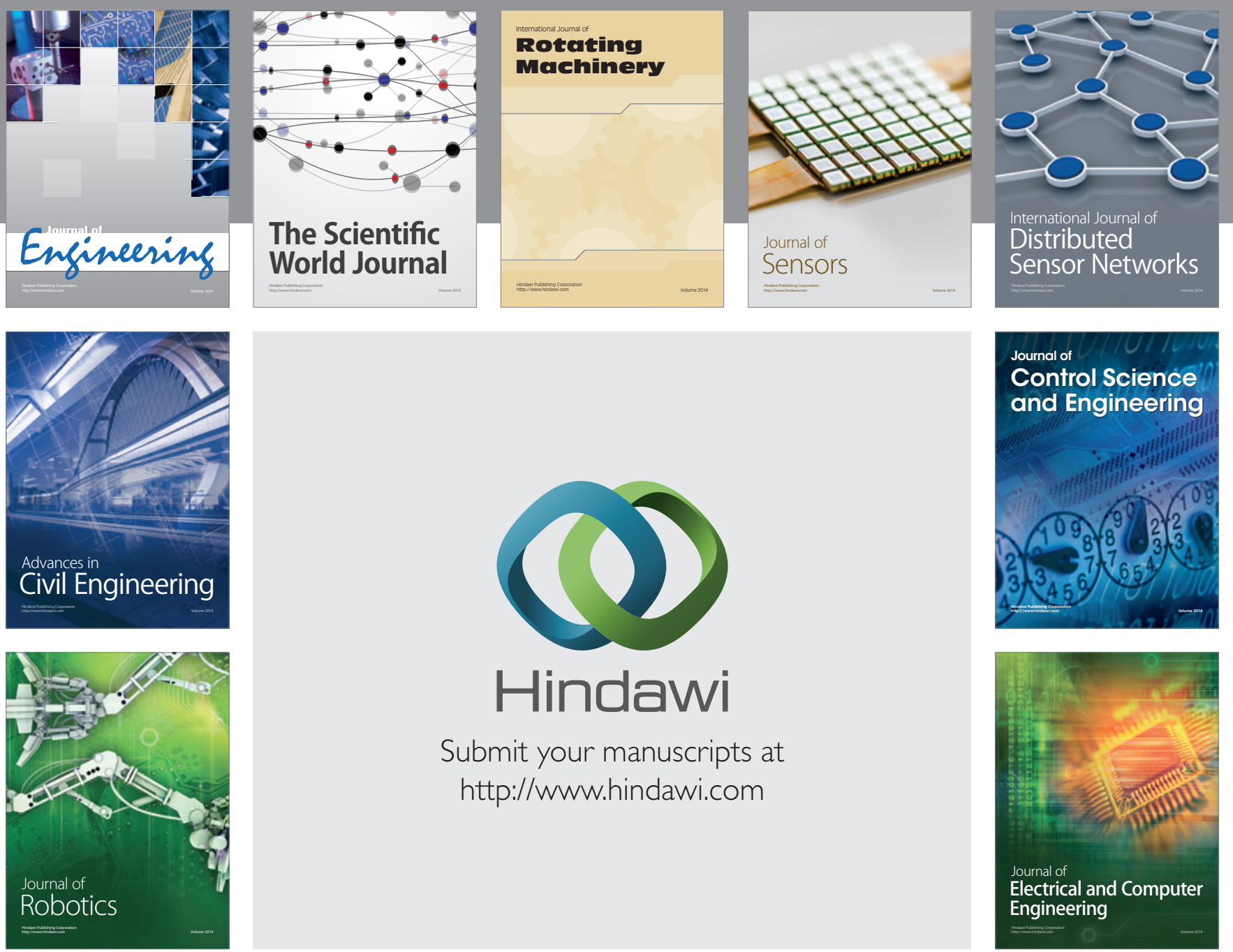

Submit your manuscripts at

http://www.hindawi.com
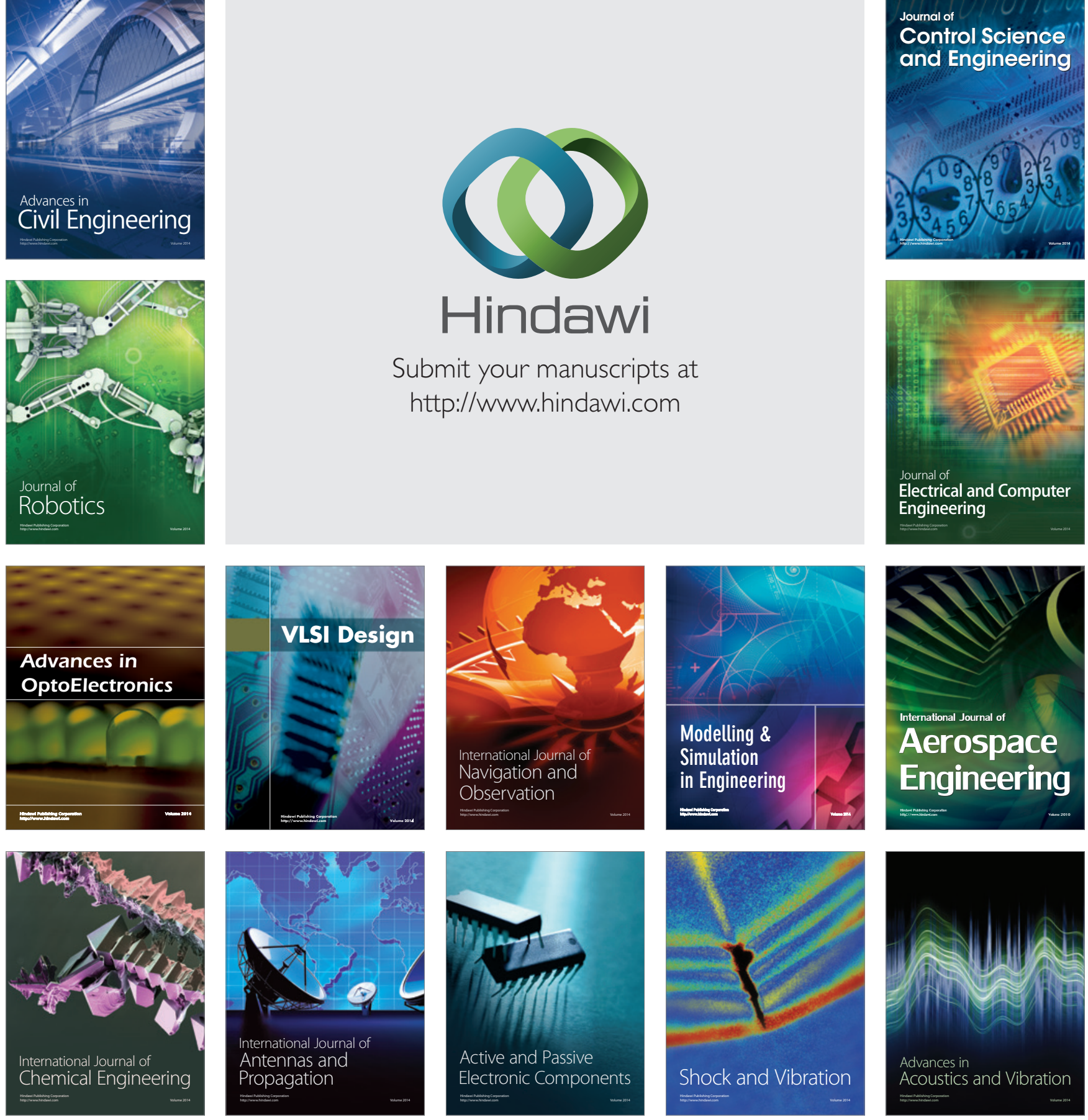